\title{
Use of Permanent Markers for Intraoperative Marking in Body Contouring Surgery: An Innovative Technique
}

\author{
Shabeer Ahmad Wani ${ }^{1 *}$, Loai Abdullah Al Salmi' ${ }^{1}$ Ovais Habib ${ }^{1}$, Mir Uzair Ul Haq ${ }^{2}$
}

1. Section of Plastic Surgery, King Fahad Medical City, Riyadh, Saudi Arabia;

2. Medical intern SKIMS Medical College, Srinagar, Kashmir, India

*Corresponding Author:

Shabeer Ahmad Wani,

Department of Plastic Surgery,

King Fahad Medical City,

Riyadh, Saudi Arabia.

Email: wanidrshabir@rediffmail.

Received: May 27, 2017

Revised: November 18, 2017

Accepted: December 28, 2017

\section{DEAR EDITOR}

Body contouring surgery after massive weight loss is becoming very common all over the world and more so in our part of world. The contouring procedures need an extensive preoperative planning and correct marking of the incisions and areas of resection. The marking fades sometimes after scrubbing the patient and use of tumescent fluids. Intraoperative precise marking can be done by use of sterile surgical markers (Figure 1). The scarcity of ink in these markers is frustrating and sometimes we need to open multiple markers, which is not cost effective. Another alternative is to use methylene blue with a Q-tip applicator. The marking by this method is not precise. We have been using Sharpie (Atlanta, Georgia, USA) colored permanent markers in a sterile pouch made from draping sheets. Circulating nurse decontaminates the outer surface of pen with an alcohol

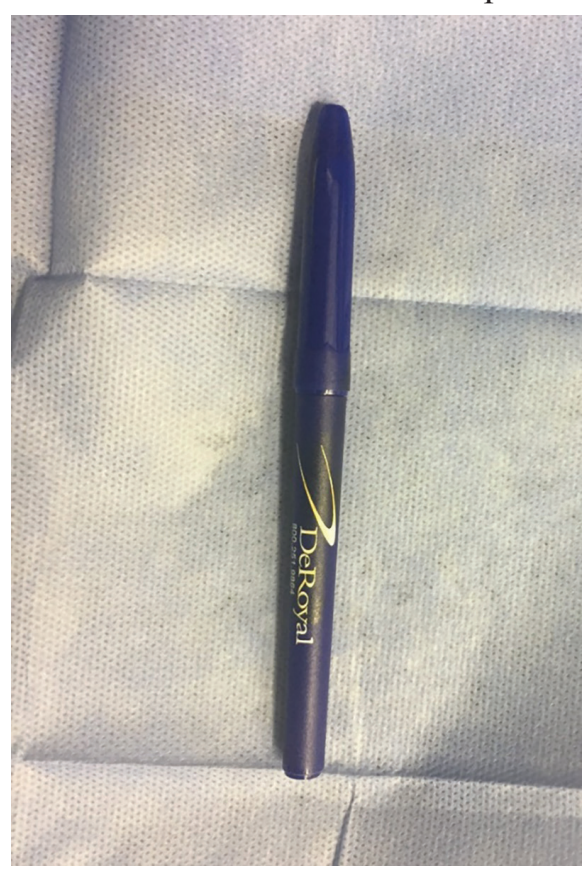

Fig. 1: Intraoperative precise marking done by use of sterile surgical markers.

swab and cap of pen is removed.

The circulating nurse hands over the pen to scrub nurse who puts it in a pouch made from sterile drape. Putting steri strips over it tightens the pouch (Figure 2). The writing tip of this marker is sterile by itself as it contains isopropyl alcohol. The antibacterial action of the writing tip has been proven in 


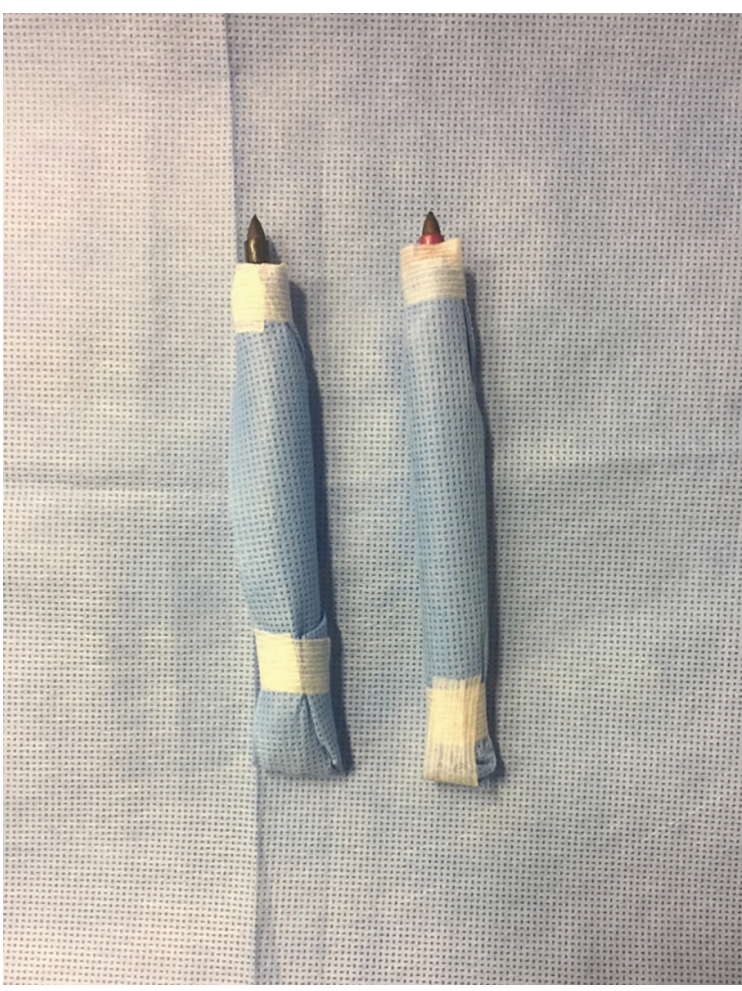

Fig. 2: The circulating nurse hands over the pen to scrub nurse who puts it in a pouch made from sterile drape. Putting steri strips over it tightens the pouch.

a research done at university of Alberta. ${ }^{1}$ At the end of the procedure the pen is removed from the pouch and recapped, and can be used in this way for multiple times. This method is cost effective, and we have an option of using multiple colors. Moreover, the Sharpie markers have ink, which dries on the surface of patient instantaneously and marks don't fade. We did not notice any infection in these patients, which could have been related to use of these markers.

\section{CONFLICT OF INTEREST}

The authors declare no conflict of interest.

\section{KEYWORDS}

Sharpie permanent; Marker; Intraoperative Marking

Please cite this paper as

Wani SA, Al Salmi LA, Habib O, Uzair Ul Haq M. Use of Permanent Markers for Intraoperative Marking in Body Contouring Surgery: An Innovative Technique. World J Plast Surg 2018;7(3):387-388. doi: $10.29252 /$ wips.7.3.387.

\section{REFERENCES}

1 Brown M. X marks the spot: Sharpies get thumbs-up for marking surgery sites. Folio 2008;46:2008. 